\title{
Risk assessment of heritage structures endangered by fluvial floods
}

\author{
M. Holicky \& M. Sykora \\ Klokner Institute, Czech Technical University in Prague, Czech Republic
}

\begin{abstract}
Floods have recently caused significant economic losses and affected a number of civil engineering structures in many countries including the Czech Republic. Various protective measures have been considered to reduce adverse consequences of flooding in future. Decisions concerning expensive measures should be preferably based on risk optimisation, taking into account cost of measures, potential societal and economic consequences and losses of cultural and heritage values in case of historic structures. Methods of probabilistic risk optimisation seem to provide a suitable tool for identification of the most effective permanent and temporary measures. Present paper provides general framework of the risk assessment of heritage structures endangered by fluvial floods. The total consequences may be assessed using Bayesian networks supplemented by decision and utility nodes. Further investigations of relevant input data including societal and economic consequences and losses of cultural and heritage values are desired.
\end{abstract}

Keywords: risk assessment, heritage structures, fluvial floods.

\section{Introduction}

Numerous regions of the Czech Republic suffered from extreme fluvial flooding in August 2002. Most existing flood protections were overwhelmed and damage exceeded 3 billion EUR. Emergency measures hastily applied during the flooding included protective barriers, prompt removal of floating debris from bridges, additional anchors for river traffic and movement of people away from endangered areas. Despite these measures, construction works were considerably damaged. Particularly severe consequences were observed in the city of Prague 


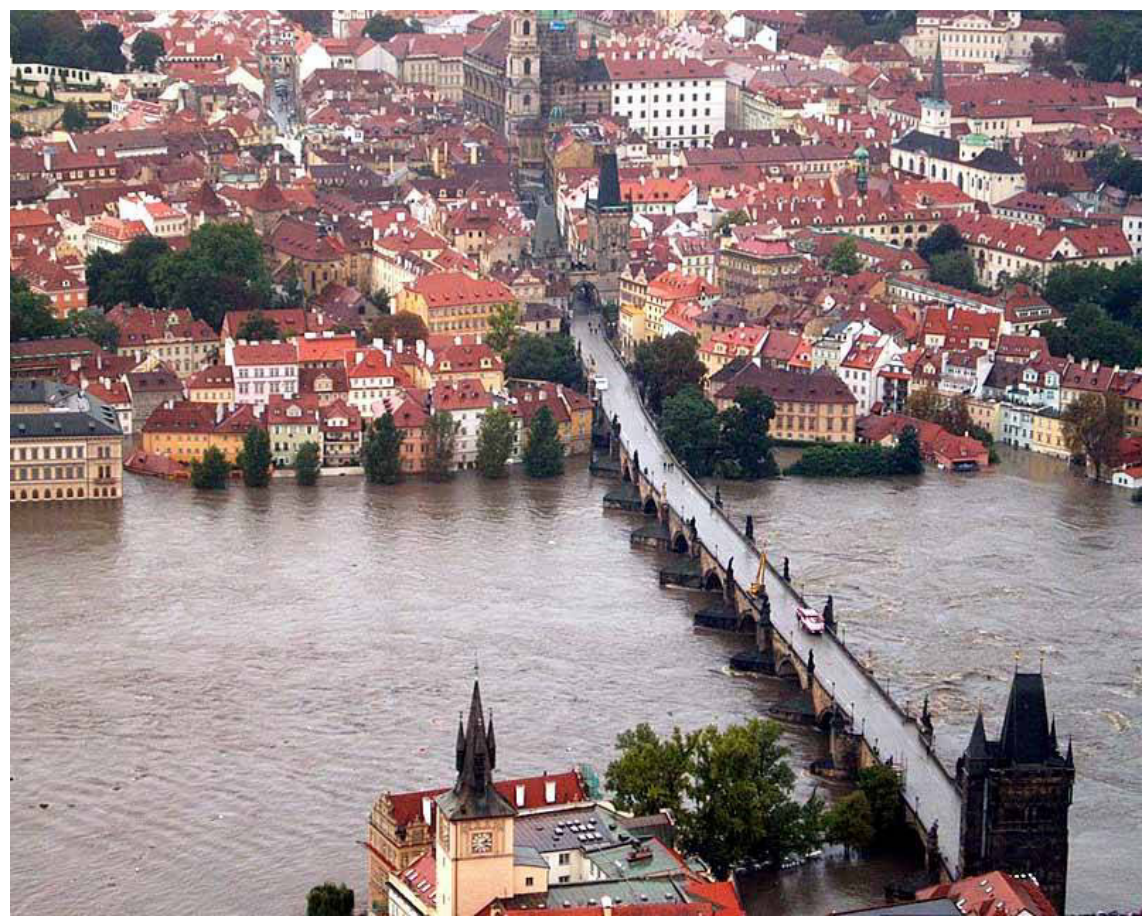

Figure 1: $\quad$ The Vltava River in Prague during the flooding in 2002.

where the Vltava River reached its historical maximum. Figure 1 shows the flooding in the city's centre, registered in the World Heritage List.

Hundreds of flooded structures included historic structures (churches, synagogues and bridges), public buildings (schools, office buildings and hotels) and residential houses. Extensive investigations of the damage provided valuable information on flood actions and causes of structural damage. Erosion, hydrostatic and debris actions were identified as principal flood actions on structures; in most cases combinations of the flood actions occurred. Main causes of structural damage included geotechnical aspects, inadequate structural properties and insufficient communication among responsible authorities, (Holicky and Sykora [1]).

The flooding had even more adverse effects in interiors of the heritage buildings (long-term increase of residual moisture with biological attacks and unfavourable drying effects). Kundzewicz et al. [2] recognized fluvial flooding as a major hazard in the Central Europe region.

In accordance with Stovel [3] effective protection of cultural heritage endangered by floods requires advance planning and preparation, taking into account landscape, structures and their contents. After the flooding responsible authorities have considered various permanent and temporary protective measures to reduce consequences of flooding in future. Decisions concerning these measures should be preferably based on assessment of flooding risk, taking 
into account potential societal and economic consequences of flooding and foreseen losses of cultural heritage values.

In the present paper general framework of risk assessment of heritage structures endangered by floods is proposed using Bayesian (causal) network, considering experience from the investigations in the Prague heritage site.

\section{General framework of probabilistic risk assessment of potentially flooded heritage structures}

The flooding in 2002 showed vulnerability of the Prague heritage site to unfavourable effects of flooding. Hence, appropriate measures should be applied to reduce adverse consequences of future floods concerning not only human health and cultural heritage, but also environment, infrastructures etc. The United Nations Disaster Relief Organisation [4] issued general guidelines for the risk assessment related to natural disasters. More recently, the European Directive 2007/60/EC [5] established a framework of the assessment and management of flood risks. Flood hazard and risk maps are to be provided within present development of flood risk management plans. Note that all the cited documents, however, do not include cultural heritage issues as follows from the critical study of the European parliament by Drdacky et al. [6].

The report of UNESCO [4] recommends expressing risks in monetary terms, although it may be difficult particularly in case of cultural heritage (Canuti et al. [7]). At present risk assessments of heritage assets are usually based on the combination of quantitative and qualitative criteria and likelihoods of unfavourable events and expected consequences are evaluated in risk rating matrices.

General framework of the risk assessment of heritage structures endangered by floods is thus proposed to provide a rational basis for decision-making concerning protective measures. Generalization of proposed methods for the assessment of other heritage assets or whole heritage sites exposed to various hazard situations is foreseen.

In general, risk analysis aims at consideration of all possible events together with their unfavourable consequences. Such events are often caused by extreme hazards including flooding. Adequate hazard scenarios and relevant occurrence probabilities need to be estimated (Stewart and Melchers [8] and Melchers [9]), often on the basis of expert judgements. Risk of various technical systems in hazard situations may then be analysed using the standard techniques such as the Event trees, Fault trees, Cause-consequence method and Bayesian (causal) networks (see Stewart and Melchers [8]). Several authors including Stewart and Melchers [8], Melchers [9] and Holicky [10] recognised that particularly Bayesian networks, supplemented by decision and utility nodes (see Jensen [11]) provide an effective tool for the risk analysis. The present study attempts to apply Bayesian networks in the risk assessment of heritage structures.

Figure 2 shows the proposed Bayesian network consisting of:

- The chance nodes Flow, Flood action, Structural damage, Geotechnical conditions, and Structural properties, 


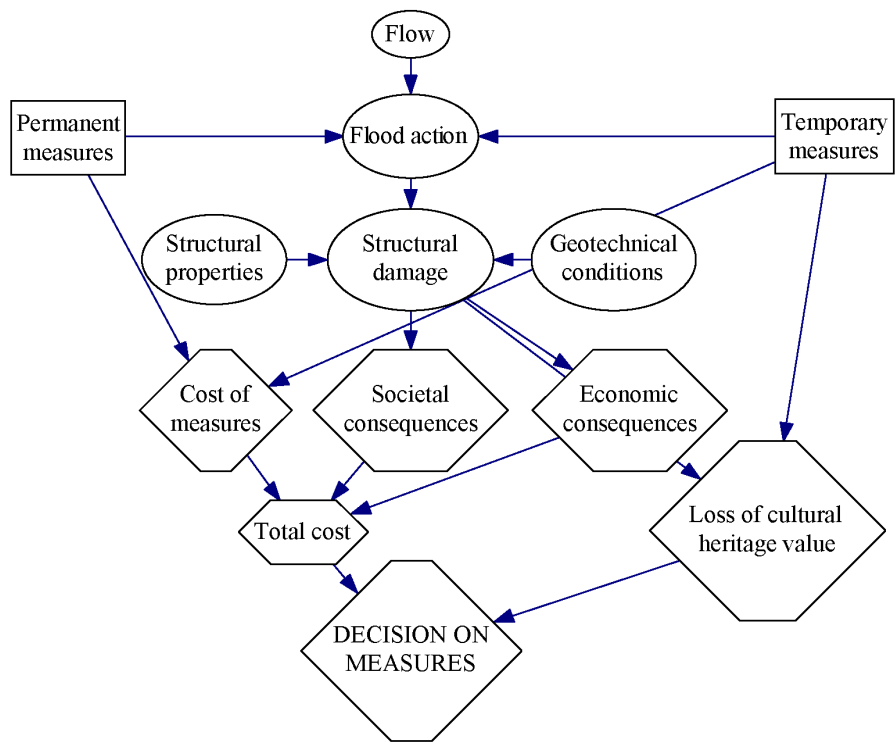

Figure 2: $\quad$ Bayesian network.

- The decision nodes Permanent and Temporary measures,

- The utility nodes Cost of measures, Societal and Economic consequences, Total cost, and Loss of cultural heritage value.

Directional arrows, interconnecting all the nodes, indicate the causal links between parent and children nodes. Note that the network is significantly simplified. In practical applications each random node may represent a subsystem and additional utility nodes may be included.

\section{Flow and flood actions}

The chance node Flow describes flows for a relevant type of flood (fluvial, flash, urban floods, floods from the sea in coastal areas etc.). According to the directive 2007/60/EC [5], floods with a low occurrence probability (an expected return period $>>100$ years), floods with a medium occurrence probability (an expected return period $\geq 100$ years) and floods with a high probability (an expected return period $<100$ years) shall be distinguished in flood risk maps. Apparently, decisions concerning protection of endangered areas need the probabilistic model for prediction of floods.

Contrary to common construction works, heritage structures may be severely affected even by floods with lower flows. Minor flows may directly lead to a loss of a cultural heritage value; for instance increased moisture may cause damage to fittings, furnishings, collections, libraries, archival records etc. Appropriate temporary measures may, however, prevent or reduce the losses. 
Prediction of floods is usually based on statistical fitting of theoretical distributions to available hydrologic data. Up to now many probabilistic models have been suggested, but none has been accepted universally. As a rule "goodness-of-fit" tests are used to identify a suitable probabilistic model and standard statistical techniques such as the method of moments or method of maximum likelihood are applied to estimate parameters of a distribution.

Pearson III distribution with lognormal transformation of data (log-Pearson III) for long-term prediction of floods is recommended in the report of IACWD [12]. Gumbel and lognormal distributions are also widely used (see Holicky et al. [13]). In general appropriate models should be selected on the basis of the statistical tests and experience with distributions of flows at other (similar) localities.

It is emphasised that statistical analysis of available hydrological data may not always be sufficient. In many cases non-statistical influences that may have developed during the period covered by the measurements should be considered. These may include:

- River management (maintenance and/or restoration of floodplains, modifications of depth, width and roughness of a river channel and removal of vegetation),

- Local paved areas affecting local flood conditions,

- Effects of deforestation, changes in land use and other human-made interventions in environment,

- Climate changes etc.

In Figure 2 the chance node Flood actions cover various actions that may occur within flooding, including hydrostatic pressure (lateral pressure and capillary rise), hydrodynamic pressure (related to velocity or waves), erosion and scour, buoyancy, debris (static, dynamic and erosion), and non-physical (chemical and biological) actions, for more details see Kelman and Spence [14]. The flood actions depend on the flow and the two decision nodes - Permanent and Temporary measures.

\section{Protective measures}

Permanent measures may include:

- Protective barriers (levees),

- River management (diversions, maintenance and/or restoration of floodplains, modifications of depth, width and roughness of a river channel),

- Arrangements improving structural properties (reinforcing structures, strengthening joints, protection of exposed openings etc.).

The first two measures that influence the flood actions are usually applied to the whole (heritage) site while the arrangements are used for a particular structure to decrease its vulnerability to unfavourable effects of floods.

Protective barriers, property relocation, immediate removing floating debris from bridges, additional anchors for river traffic, movement of people and movable heritage properties away from endangered areas may then be considered 


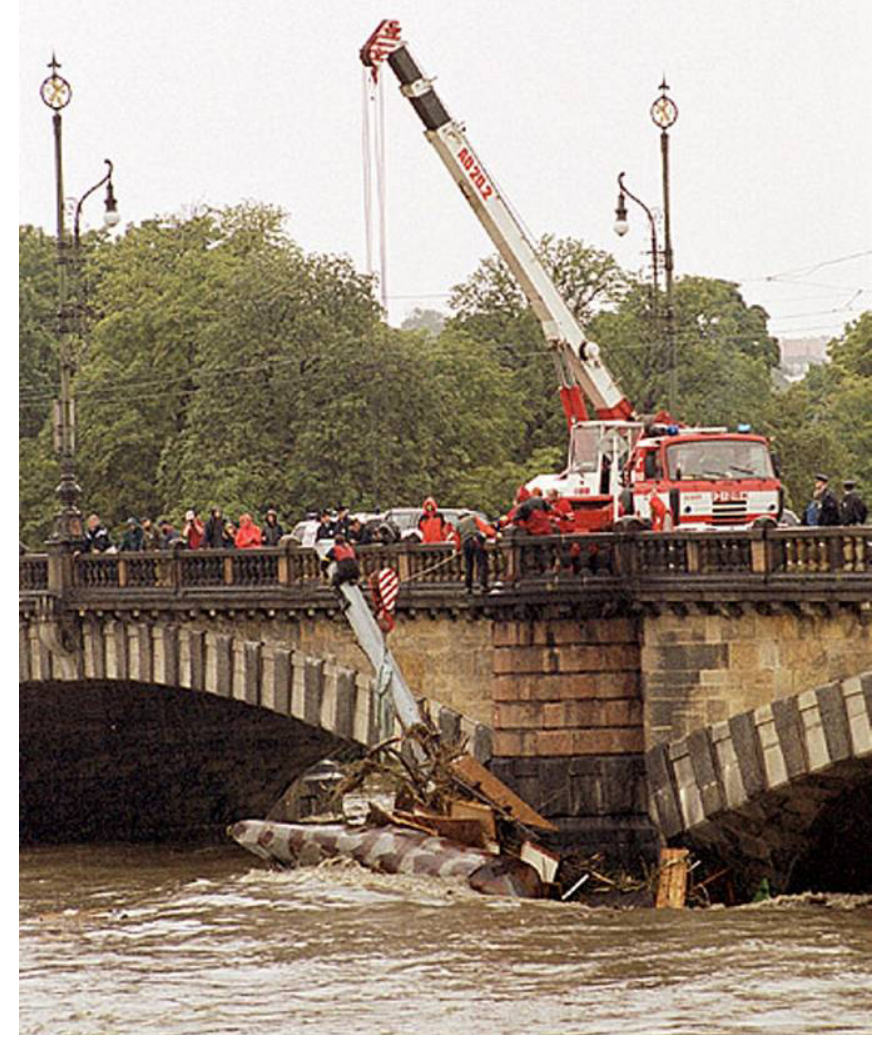

Figure 3: Accepted temporary measure - crane removing floating debris from bridge piers

as the temporary measures. Figure 3 shows the example of the temporary measure accepted within the flood in 2002 - a crane removing floating debris from bridge piers. The utility node Cost of measures describes expected expenditures on the permanent and temporary measures.

\section{Assessment of consequences}

\subsection{Structural damage}

The flood actions may lead to structural damage (random node in the network) covering structural malfunctioning due to local failures, partial or total collapse. Probability of structural damage and its extent depends on:

- Geotechnical conditions (foundation, underground water level and flows, propagation of caverns),

- Structural properties (structural integrity, sensitivity of construction materials to increased moisture, resistance of openings). 
Basis of reliability analysis of heritage structures is provided in an annex to the international standard on the assessment of existing structures (ISO 13822 [15]). The annex is based on the fundamental principles provided in the documents of the International Council on Monuments and Sites such as [16].

\subsection{Societal and economic consequences}

Structural damage may result in societal and economic consequences and loss of a cultural heritage value. The utility node Societal consequences describes the expected societal consequences dependent on the expected number of fatalities due to the structural collapse (see e.g. Trbojevic [17]) and an acceptable compensation cost for a fatality, which may be approximated by the so-called societal value of statistical life as proposed by Holicky [10].

Economic consequences primarily describe cost of repairs of a structure damaged by flooding and cost related to structural malfunction. Note that for a reference period considered in risk analysis, discount rate should be taken into account to express the expenses during this period in current costs, Holicky [10].

\subsection{Loss of cultural heritage value}

The loss of a cultural heritage value of a structure and its content is usually estimated by an expert judgement. In accordance with ISO 13822 [15], the cultural heritage value includes authenticity and integrity of a historic structure and its character-defining elements (historic materials, forms, locations, spatial configurations, morphology, concept and details, structural design, etc.). Note that the loss of cultural heritage value may be perceived as significant societal and economic consequences. However, it may be appropriate to consider the loss of cultural heritage value and economic and societal consequences separately, particularly when the loss of cultural heritage value is assessed only qualitatively.

Several methods have been proposed for the assessment of an environmental value of assets, which may be a similar issue to the estimation of the cultural heritage value as indicated by Bedate et al. [18] and Sanz et al. [19]. Alternatively, qualitative assessment of the cultural heritage value may be applied. ISO 13822 [15] indicates that judgments about the cultural heritage value may differ from culture to culture and thus it is difficult to establish any fixed criteria.

\section{Decision-making}

Decisions on the protective measures should be based on optimisation of the total cost including the loss of the cultural heritage value. When the cultural heritage value is assessed qualitatively, the loss of the value and total cost are considered separately.

Apparently, the effective optimisation will in turn facilitate proposals of flood-protection strategies appropriate for heritage structures, their contents and occupants. Collaboration of various professionals in the construction industry 
and experts on heritage assets including engineers, architects, surveyors, planners, archaeologists, historians with responsible municipal and national authorities may be highly beneficial in this regard.

\section{Concluding remarks}

Analysis of consequences of the fluvial flooding in 2002 in the Prague heritage site reveals that main causes of structural damage can be subdivided into geotechnical aspects, inadequate structural properties and insufficient communication among responsible authorities. Decisions concerning protective measures should be preferably based on risk optimisation, taking into account potential societal and economic consequences and foreseen losses of cultural heritage values. Bayesian networks supplemented by decision and utility nodes provide an effective tool for the risk analysis. Judgment about the cultural heritage value is one of the most difficult issues within the risk assessment of heritage structures. It should be taken into account that heritage structures may be severely damaged even by minor flows causing increased moisture in interiors.

\section{Acknowledgements}

This study has been conducted at the Klokner Institute, Czech Technical University in Prague, within framework of the research project Assessment of historical immovables A/CZ0046/2/0013, supported by a grant from Iceland, Liechtenstein and Norway through the EEA Financial Mechanism, the Norwegian Financial Mechanism and the Czech state budget.

\section{References}

[1] Holicky, M. \& Sykora, M., Forensic investigation of fluvial flood damage in the Czech Republic. Proceedings of ICE Civil Engineering, 162(May 2009), pp. 33-37, 2009.

[2] Kundzewicz, Z.W., Ulbrich, U., Brücher, T., Graczyk, D., Krüger, A., Leckebusch, G.C. et al, Summer floods in central Europe: Climate change track? Natural Hazards, 36(1-2), pp. 165-189, 2005.

[3] Stovel, H., Risk preparedness: a management manual for world cultural heritage, International Centre for the Study of Preservation and Restoration of Cultural Property: Rome, pp. 145, 1998.

[4] UNESCO-UNDRO, Natural disasters and vulnerability analysis, Office of the United Nations Disaster Relief Co-ordinator: Geneva, pp. 49, 1979.

[5] The European parliament and the Council, Directive 2007/60/EC on the assessment and management of flood risks, Official Journal of the European Union: Brussels, pp. 8, 2007.

[6] Drdacky, M., Binda, L., Herle, I., Lanza, L.G., Maxwell, I. \& Pospisil, S., Protecting the Cultural Heritage From Natural Disasters (study of the 
European Parliament IP/B/CULT/IC/2006_163, PE 369.029), The European Parliament: Brussels, pp. 100, 2007.

[7] Canuti, P., Casagli, N., Catani, F. \& Fanti, R., Hydrogeological hazard and risk in archaeological sites: some case studies in Italy. Journal of Cultural Heritage, 1(2), pp. 117-125, 2000.

[8] Stewart, M.G. \& Melchers, R.E., Probabilistic Risk Assessment of Engineering Systems, Springer: Berlin, pp. 288, 1997.

[9] Melchers, R.E., Structural Reliability Analysis and Prediction, John Wiley \& Sons Ltd.: Chichester, England, pp. 437, 2001.

[10] Holicky, M., Probabilistic risk optimization of road tunnels. Structural Safety, 31(3), pp. 260-266, 2009.

[11] Jensen, F.V., Introduction to Bayesian Networks, Springer: Berlin, pp. 178, 1997.

[12] IACWD (US Interagency Advisory Committee on Water Data - Hydrology Subcommittee), Guidelines for determining flood flow frequency, US Dept. of the Interior, Geological Survey, Office of Water Data Coordination: Reston, Virginia, 1983.

[13] Holicky, M., Jung, K. \& Sykora, M., Assessment of Extreme Discharges of the Vltava River in Prague. Proceedings of the First International Conference on Flood Recovery, Innovation and Response FRIAR 2008, eds. D. Proverbs, C.A. Brebbia \& E. Penning-Rowsell, WIT Press: Southampton, pp. 105-112, 2008.

[14] Kelman, I. \& Spence, R., An overview of flood actions on buildings. Engineering Geology, 73(3-4), pp. 297-309, 2004.

[15] ISO 13822, Bases for design of structures - Assessment of existing structures. Annex I Heritage structures, TC98/SC2/WG6: Geneve, Switzerland, pp. 10, 2008.

[16] ICOMOS, Recommendations for the analysis, conservation and structural restoration of architectural heritage, International council on monuments and sites: Paris, pp. 37, 2003.

[17] Trbojevic, V.M., Another look at risk and structural reliability criteria. Structural Safety, 31(3), pp. 245-250, 2009.

[18] Bedate, A., Herrero, L.C. \& Sanz, J.Á., Economic valuation of the cultural heritage: application to four case studies in Spain. Journal of Cultural Heritage, 5(1), pp. 101-111, 2004.

[19] Sanz, J.Á., Herrero, L.C. \& Bedate, A., Contingent Valuation and Semiparametric Methods: A Case Study of the National Museum of Sculpture in Valladolid, Spain. Journal of Cultural Economics, 27(3-4), pp. 241-257, 2003. 